\title{
DOBLE CORAJE, LAS PIONERAS DE LA TRADUCCIÓN
}

\author{
DOUBLE COURAGE, WOMEN AS TRANSLATION PIONEERS
}

Valentina Tomassini

\section{Resumen:}

En muchas teorías traductológicas se ha evidenciado un paralelismo entre el estatus del texto traducido, considerado inferior a la obra original, y el de las mujeres, subestimadas tanto en la sociedad como en la literatura. Por lo tanto, en las últimas décadas, la perspectiva de género se ha ocupado de rescatar el trabajo de las traductoras y de de-construir una teoría que no había incorporado a las mujeres en el proceso de estudio del fenómeno y en la reflexión crítica. En concreto, en este artículo pretendemos dar una muestra dela innovación que las mujeres han aportado en este campo y de las estrategias que han llevado a cabo para subrayar su identidad en el texto. Debido a la centralidad del mundo anglonorteamericano en las especulaciones de género sobre traducción, nuestro recorrido se fundamenta sobre todo en el trabajo de las investigadoras $\mathrm{y}$ traductoras canadienses, para luego abrirse a un breve estado de la cuestión acerca de la recepción que su pensamiento ha tenido en el contexto español.

\section{Palabras clave:}

Traducción feminista canadiense, estudios de traducción, género y traducción, traductoras.

\section{Abstract:}

Many translation theories pointed out a similarity between the status of the translated text, which was considered as inferior to the original, and the women's one, because of their underestimation both in society and literature. For this reason, during the last decades, gender perspective has tried to recover translatress'works and to deconstruct a theory that hadn't involved women in its studies on the translation process. In particular, in this article we would like to show women's innovation in this field and the strategies they have created to underline their identity in the text. Because of the importance of Anglo-American theories about gender and translation, our work is primarily based on feminist translation school in Canada, in order to analyse, then, its reception in the Spanish context.

\section{KEYWORDS:}

Canadian feminist translation, translation studies, gender and translation; translatresses. 


\section{INTRODUCCIÓN}

El presente estudio se propone destacar la innovación llevada a cabo por las feministas canadienses en el campo de las teorías sobre traducción. Para ello, hemos dividido nuestro artículo en dos partes: en la primera, se describe en general la posición de las mujeres traductoras antes de la segunda mitad del siglo XX y se intenta esbozar una primera matriz teórica de los estudios traductológicos feministas, mientras que, en la segunda parte, nos hemos fijado en los pasos teóricos que las "pioneras" canadienses han dado para la recuperación del trabajo de sus predecesoras y para la elaboración de algunas estrategias que visibilizaran su identidad en los textos traducidos. En concreto, el proceso de re-construcción y re-ubicación de las mujeres en el mundo de la traducción sigue un recorrido gradual, que va de la teoría a la práctica. Por lo tanto, hemos intentado dar una muestra de ello presentando, en primer lugar, la novedad de sus concepciones y, en segundo lugar, los recursos aplicativos desarrollados, pasando por los cambios asociados a la representación simbólica del proceso traductivo (metáforas, mitos, léxico, etc.). Por último, hemos comprobado qué recepción ha tenido la teoría traductológica de las feministas canadienses en España trazando algunos breves perfiles de las "pioneras" de aquel contexto, es decir las primeras estudiosas españolas que divulgaron el nuevo pensamiento sobre traducción vinculado con el género. A nivel metodológico, nos hemos apoyado en los postulados del deconstruccionismo, en que se basan también las teorías feministas analizadas: puesto que un texto siempre es un tejido en que se entrecruzan muchos otros, no vamos a centrarnos en un estudio en concreto, sino que intentaremos poner de relieve dicho tejido a nivel grupal, extrapolando algunos hilos comunes a todas las especulaciones sobre el tema. Claramente, nuestro enfoque ha sido conjugado con la ginocrítica, por su voluntad de encontrar "la diferencia" de estas mujeres y, en general, con las varias teorías que las traductoras canadienses han incorporado a sus contribuciones.

\section{EL TÉRMINO “PIONERAS”}

Si buscamos la palabra "pionero, ra" en el Diccionario de la Real Academia Española encontramos las siguientes definiciones aplicables a nuestro caso: (1) "persona que inicia la exploración de nuevas tierras" y (2) "persona que da los primeros pasos en alguna actividad humana". Por lo tanto, según esta última acepción, las pioneras de la traducción deberían ser las que, por primera vez, se pusieron a traducir textos y, por consiguiente, a reflexionar sobre esa práctica, de manera más o menos sistemática. Sin embargo, debido al intento de ocultamiento de toda creación intelectual femenina por parte del patriarcado, el trabajo de las primeras traductoras ha llegado de una forma muy incompleta y fragmentada. En especial, la presencia de un autor del texto traducido favoreció aún más el proceso de desvaloración de las traductoras: su obra 
quedó como sumergida bajo el nombre del autor que estaban traduciendo. Aunque tampoco los traductores tenían mucha visibilidad hasta el siglo $\mathrm{XX}$, es evidente que, por razones histórico-sociales relacionadas con la condición de las mujeres, la marginalización de la traducción afectó doblemente a las traductoras, limitando así la accesibilidad de su legado. Justo en la segunda mitad del siglo XX, cuando empezó a visibilizarse la traducción como actividad de escritura "paritaria", algunas mujeres iniciaron la exploración de una nueva tierra, es decir la "isla" que guardaba el trabajo de estas "pioneras silenciadas" y, más en general, el punto de vista dialógico y femenino sobre traducción, dentro del "mar" del monólogo masculino. Entonces, la innovación que vamos a presentar atañe más a la primera acepción del término "pioneras": las feministas canadienses fueron las primeras en sondear críticamente el terreno de la traducción desde lo femenino hasta revindicar su papel de lectoras/escritoras. De esta forma, recuperaron el camino trazado a escondidas por sus predecesoras, las "pioneras silenciadas", y lograron transformar la traducción en un proceso performativo de construcción de significado, que incorporara la pluralidad y la diferencia. Para entender el alcance de esta ruptura con el pasado y con el presente de algunas teorías traductológicas contemporáneas, primero sería necesario describir en grandes líneas cuál era la posición de las mujeres con respecto a la traducción y su historia hasta el surgimiento de la escuela canadiense.

\section{UBICAR A LAS MUJERES EN LA TRADUCCIÓN}

Es un hecho notorio que la traducción tiene un largo pasado pero una historia corta: aunque su carácter pragmático y funcional la ha llevado a desarrollarse ya en tiempos remotos, los estudios académicos y la reflexión sistemática sobre el tema han surgido solo en los últimos setenta años. Frente a esta discrepancia entre la práctica y la teoría, incrementada por los prejuicios sobre la mera instrumentalidad del texto traducido, llama la atención la parcial equidad de roles entre traductoras y traductores. Aunque no se trate de una igualdad numérica ni mucho menos de una misma visibilidad, podríamos afirmar que la actividad que se estaba llevando a cabo no suponía muchas distinciones entre los $\operatorname{sexos}^{1}$ y que el trabajo de un traductor planteaba casi los mismos retos que el de una traductora. Presumiblemente, eso fue posible gracias a la marginalidad de la traducción, desde siempre considerada como una forma de escritura colateral e inferior a la propia creación, que resultó ser un puente para que otras marginalizadas -las mujeres en este caso- pudieran acceder a la cultura de su época y contribuir a la difusión de nuevas ideas. Si la escritura femenina se ahogaba muchas veces en las prohibiciones patriarcales, la escritura traducida, en cambio,

1 Una de las más importantes consistía en el vedo de traducir la Biblia para las traductoras. Para una historia detallada de la traducción de la Biblia en el marco de los estudios feministas, véase: Simon, 1996, pp. 105-126. 
constituía una herramienta para respaldar a los hombres: con ella de hecho las mujeres no se expresaban, sino que colaboraban al refuerzo y a la divulgación de la producción masculina en otros contextos (Delisle, 2002, p. 7). En realidad, las mujeres podían aprovechar la ocasión para poner de lo suyo en un texto que compartía su misma condición subordinada: mirando hacia atrás, a la luz de las concepciones modernas, nos damos cuentas de que en este espacio lo femenino pudo encontrar su lugar socialmente aceptado. Paradójicamente, las mujeres y la traducción estaban al margen del discurso patriarcal dominante y, sin embargo, estaban en el centro de transmisión de las representaciones simbólicas de su sociedad. Al igual que las escritoras y sus textos, las traductoras no eran "transportadoras neutras" de mensajes ya formulados, sino que construían sus traducciones de acuerdo con sus interpretaciones y estilo, hasta hacerse "autoras", poniendo marcas de su presencia silenciada en el texto. ${ }^{2}$ Al mismo tiempo, estas mismas mujeres desarrollaban tácitamente su pensamiento acerca de su actividad, se interrogaban sobre las operaciones que cumplían y, por eso, empezaron a echar las bases para una primera reflexión teórica. Aunque, como hemos dicho, haya llegado muy poco de esta teoría embrionaria, ${ }^{3}$ a lo mejor fragmentada en muchos paratextos, no se puede negar que el aporte mujeril a la traducción ha sido consistente y en su mayoría ocultado. Solo a partir de los años ochenta, la perspectiva de género se ha esmerado para poder corregir esta aparente marginalidad en una centralidad efectiva, que recupere el trabajo de las traductoras del pasado y valorice la innovación de las del presente. Este giro se sitúa en una tendencia dignificadora más general y diríamos casi "concéntrica": mientras las mujeres traductoras reivindicaban su posición en el texto traducido, la traducción, por su parte, empezaba a abrirse camino en el centro del canon literario. Mujeres y traducciones, las que, en un cuadro discursivo androcéntrico, habían sido objetos de una asociación constante basada en su marginalidad, ${ }^{4}$ finalmente intentaban salir juntas de las constricciones patriarcales. Por lo tanto, nuestro estudio se propone averiguar, por un lado, cuáles han sido los pasos teóricos -todavía inacabados- para la recuperación del trabajo femenino en traducción y, por otro, las estrategias que las mismas traductoras han aplicado para visibilizar su identidad textual y lograr finalmente una posición central en su escritura.

\section{APUNTES SOBRE EL ORIGEN DE LOS ESTUDIOS FEMINISTAS DE TRADUCCIÓN}

2 En línea con la equivalencia individuada por Lefevere entre traducción y reescritura (1992).

3 Algunos trabajos de recuperación del trabajo de las traductoras se han llevado a cabo: cfr. Agorni, 2014; Delisle, 2002; Guerrero Alcalde, 2017; Pieretti, 2002 y Romero López, 2015 y 2016.

4 Acerca del léxico sexista empleado en el ámbito de la traducción, véase: Godayol, 2008, pp. 6769. 
Al cabo de muchos siglos de subordinación del binomio traducciones-mujeres, la primera huella de un cambio se encuentra paradójicamente en la especulación de un hombre, Jacques Derrida. En sus obras, el filosofo francés desarrolla una progresiva deconstrucción de las categorías rígidas en que habían quedado atrapados los dos conceptos en relación. Para ello, sus argumentaciones se apoyan en el punto de contacto más evidente entre textos y mujeres, es decir su vinculación con el género (Derrida, 1986). De hecho, esta misma palabra se aplica tanto al género textual como al sexual, entendido como "la definición cultural del comportamiento que se define como apropiado a cada sexo dentro de una sociedad determinada y en un momento determinado. El género es un conjunto de papeles sociales. Es un disfraz, una máscara, una camisa de fuerza dentro de la cual hombres y mujeres practican una danza desigual" (Lerner, 1990, p. 339). En ambos casos, Derrida observa que la ley del género intenta ordenar la diferencia impura que se da en la realidad en unas categorías normadas, limitadoras y, por lo tanto, excluyentes de toda contaminación. Las mujeres reales, ${ }^{5}$ encarnadas en cuerpos y circunstancias variables a nivel personal, político, social, etc., se alejan muy a menudo de las expectativas que se construyen sobre ellas, sin poder controlar del todo la reacción de rechazo que eso genera. La traducción como hibridación y diálogo cultural no puede funcionar en ningún caso como el género textual al que remite, ya sea solo por producirse en otro horizonte lingüístico e histórico. En el sistema conceptual occidental, estos desajustes constituyen una anomalía perturbadora, justo lo que Derrida pretende de-construir y poner en entredicho. Efectivamente, si para el filósofo el significado lingüístico reside en su misma "différance" (o sea en su diferencia ontológica con respecto a lo que el signo está representando y en la consiguiente postergación de un pleno entendimiento que dicha discrepancia presupone), está claro que las relaciones de poder entre traducción y original carecen de sentido, puesto que la supervivencia de ambos textos procede de su estricta relación de dependencia mutual. Por lo tanto, apoyándonos en la tesis de Lori Chamberlain podríamos deducir que el pensamiento de Derrida les concede implícitamente a las mujeres un papel igualitario: "By subverting the autonomy and privilege of the so-called original text, he argues for the interdependence of writing and translating - and, implicitly, against a politics of translation that depends on gender violence" (Chamberlain, 1998, p. 96). Es esta centralidad de la diferencia, en contra de toda clasificación estricta, la base de la que se despliega la nueva estética traductiva de las feministas canadienses, a partir de los años ochenta. Los factores que determinaron la acogida del pensamiento derridiano por parte de estas mujeres son por lo menos dos: por un lado, la facilidad de acceso y circulación de las ideas del filósofo francés en el contexto francófono de Canadá y, por otro lado, el contexto bicultural en sí,

5 "évite de traiter le féminin comme une puissance générale et générique, il fait sa part à l'évènement, à la performance, à l'aléa, à la rencontre" (Derrida, 1986, pp. 278-279). 
como lugar en el que las diferencias lingüísticas e identitarias suponían un esfuerzo continuo para la inclusión y el reconocimiento de los dos mayores grupos culturales del país. Encima, su actividad de reflexión y redefinición del proceso traductivo fue inevitablemente acompañada -y, en cierta medida, estimulada- por el surgimiento de actos, instituciones, documentos, conferencias y espacios académicos de debate a favor del intercambio y de la equidad entre la comunidad francesa y la inglesa (Mezei, 2013, pp. 22-25). Otra matriz determinante para el desarrollo de la escuela canadiense fue sin duda el feminismo francés:

[...] the transatlantic displacement of the writings of the French feminists, Luce Irigaray, Julia Kristeva and Hélène Cixous, into the Anglo-American intellectual world [...]. This exchange brings into light the network of tensions which are so characteristic of our current intellectual context: the conflictual pulls between internationalist feminist solidarity and national affiliations, the deconstructive drive toward attenuation of authorship and the continuing structures of textual authority, the fading of disciplinary borders and their continual reappearance (Simon, 1996, p. 4).

De entre las primeras voces que empezaron a recoger dichas influencias para transformar el panorama traductológico a la luz de las cuestiones de género destacan las de Barbara Godard (1989), Suzanne de Lotbinière-Harwood (1991), Sherry Simon (1996) y Louise Von Flotow (1997). La elaboración de su teoría feminista de la traducción procedió también de las experimentaciones deconstructivistas de las escritoras quebequesas de finales de setenta y tuvo lugar en espacios que se expandieron paulatinamente de los paratextos a las revistas, ${ }^{6}$ de las conferencias a los artículos académicos, hasta llegar a volúmenes enteros. Los primeros estudios completos que aparecieron fueron Gender in Translation. Cultural Identity and the Politics of Transmission (1996) de Sherry Simon y Translation and Gender: Translating in the 'Era of Feminism' (1997) de Luise von Flotow, dos textos que siguen siendo básicos para cualquier investigación que quiera explorar el tema. Desde entonces, la cantidad de publicaciones al respecto ha crecido de forma exponencial, ${ }^{7}$ hasta generar un campo de conocimiento que es muy difícil de definir por la gran variedad de enfoques epistemológicos y contextos lingüísticos-culturales desde los que ha sido abarcada la relación mujeres-traducción. Por lo tanto, a continuación, nos limitaremos a enfocar la cuestión desde la perspectiva de la escuela canadiense, dando por sentado que su elaboración de los principales conceptos novedosos constituyó la base de las demás aproximaciones feministas al tema de la traducción.

6 En este sentido, Tessera es una de las mayores revistas literarias feministas y bilingües, centrada en las relaciones de poder que intentan sexualizar el discurso literario y, por ende, traductivo.

7 En su Language, Gender and Feminism (2011) Mills y Mullany ofrecen una muestra de la gran cantidad de estudios al respecto en el contexto cultural de occidente. 


\section{UN NUEVO CONCEPTO DE TRADUCCIÓN Y TRADUCTOR@}

Para elaborar un nuevo concepto de traducción desde su posición marginal, las mujeres tuvieron que partir de su misma condición. La identificación simbólica ya consolidada entre su estatus y el de la traducción les permitió detectar fácilmente las subestimaciones que ambas habían sufrido a lo largo de los siglos. Por si fuera poco, en el proceso de recuperación de su trabajo como traductoras, las mujeres se encontraron en una intersección favorable y diríamos casi "metadiscursiva": hablar sobre traducción implicaba el uso de recursos aplicativos, desarrollar un pensamiento feminista en este ámbito significaba justo traducir las instancias ideológicas y políticas reales al mundo de los textos y de las operaciones transculturales. En práctica, las mujeres lograron sacar provecho de aquella analogía discriminatoria en la que habían sido relegadas. Si la traducción es un objeto de estudio secundario, como lo son las mujeres en una sociedad patriarcal, entonces ellas son las más aptas para redefinirla y actualizarla. Si es verdad que "because they are necessarily «defective», all translations are «reputed females»" (Simon, 1996, p. 1), entonces la traducción habla el idiolecto femenino y necesita un metalenguaje que se ajuste a su naturaleza y que corrobore el poder femenino de la procreación con el de la creación simbólica (el nombramiento ${ }^{8}$ ). Si la identidad femenina se ha construido hasta ahora como un signo defectivo y fragmentado (Guerra-Cunningham, 1995), entonces ella sabe reconocer lo fronterizo y la doblez de las identidades proyectadas en los textos traducidos. Brevemente, en palabras de Barbara Godard, podríamos decir que "the translator [i]s she" (Godard, 1985), queel/la traductor@ desempeña el papel tradicionalmente asociado a la feminidad de mediador@cultural,de facilitador@ de intercambio entre grupos sociales. Mientras muchas mujeres se proponían poner de relieve su estado de subordinación a través de las teorías feministas, las traductoras y las teóricas de la traducción sacaron de sí mismas un doble coraje, revindicando su identidad dentro de un sector que a su vez intentaba brotar del sistema literario dominante. Las traductoras tienen que transcender las diferencias, superar los obstáculos derivados de una posible autoría masculina ${ }^{9} \mathrm{y}$, sobre todo, afirmar la dignidad de su trabajo como escritoras. Eso no quiere decir simplemente firmar el texto o interactuar con su significado, sino también esforzarse para modificar el canon literario contemporáneo, recuperando las obras de autoras que nunca se han traducido, denunciando los casos en los que se distorsionaron textos femeninos para que encajaran con la ideología patriarcal o cuestionando el lenguaje sexista de

8 Véase: Lerner, 1990, pp. 268-270.

9 " $[\ldots]$ one of the challenges for feminist translators is to move beyond questions of the sex of the author and translator. Working within the conventional hierarchies...the female translator of a female author's text and the male translator of a male author's text will be bound by the same power relations: what must be subverted is the process by which translation complies with gender constructs" (Chamberlain 1988, p. 472). 
algunas traducciones (Castro Vázquez, 2008, pp. 288-289). Desde esta perspectiva, el/ la traductor@ pasa de ser alguien que se ocupa de reproducir un texto preexistente, según la visión tradicional, a ser parte activa del proceso de significación textual. Su actividad está destinada a reescribir el texto, en coproducción con el/la autor@, en el marco de una acción política e ideológica orientada hacia la valoración positiva de la diferencia. Las demás especulaciones desarrolladas en aquella época, como por ejemplo la teoría del polisistema de Even-Zohar, la Skopostheorie de Reiss y Vermeer o la teoría descriptiva de la traducción de Toury, aunque amplíen la mirada sobre el fenómeno traductivo más allá de lo lingüístico, no plantean un proyecto crítico militante al que ser fiel: "for feminist translation, fidelity is to be directed toward neither the author nor the reader, but toward the writing project-a project in which both writer and translator participate" (Simon, 1996, p. 2). El nuevo modelo de traductor@, entonces, consiste justo en una personalidad adicional a la del autor@, en diálogo performativo con la obra y empeñad@en un proyecto de enriquecimiento pluridimensional del texto con implicaciones identitarias y feministas. No se trata de distorsionar el contenido inicial sino de proponer una nueva interpretación, que se presente como tal, en su diferencia con respecto al texto de partida, según las coordenadas espaciotemporales en las que se despliega. Como destaca Simon, el proceso de traducción es contingente y se desarrolla en el marco de un significado necesariamente provisional y continuamente postergado a las nuevas lecturas (Simon, 1996, p. 27). La traducción ya no se concibe como una actividad determinada por su finalidad, como afirmaba la Skopostheorie (Reiss \& Vermeer, 1984), ni como un elemento que analizar para poder inducir los procesos decisionales y las normas adoptadas por el/la traductor@ (Toury, 1995). Por el contrario, contribuyendo al así llamado "giro cultural" (Mary Snell-Hornby, 1990), las feministas se muestran conscientes de sus manipulaciones dirigidas a la recuperación del papel activo de la traducción y a la exhibición de sus intervenciones subjetivas en la lengua del texto de llegada. La "invisibilidad del traductor" (Venuti, 1995) no representa una opción practicable si se mira a la traducción como a un espacio de encuentro transcultural, en el que dos culturas -con toda la diversidad que incluyenentran en contacto sin jerarquías. Este punto cobra más sentido si contextualizamos esta teoría en su "cuna" geográfica: las feministas canadienses querían requilibrar la asimetría vigente entre la mayoría anglófona y la minoría francófona, no solo a través de sus trabajos sino también entablando una cooperación asidua entre las escritoras y traductoras de ambos grupos lingüísticos. Es más, algunas de ellas habían ya superado las barreras formales entre autor@y traductor@ en el ámbito de su misma producción literaria: el bilingüismo les permitió acceder a ambos papeles contemporáneamente a través de la autotraducción. ${ }^{10}$ Para la escuela canadiense, la traducción es sobre todo un diálogo orientado hacia la interactividad y el intercambio entre dos caras -autor@/

10 Como en el caso de la escritora Nancy Houston, por ejemplo. 
traductor@ u original/traducción- de la misma medalla. Como toda relación, la traducción no busca una verdad absoluta en el texto, sino la construcción fluida de un intersticio en que convivir con el original, lejos de la normatividad de las oposiciones binarias que venían impidiendo su libre manifestación.

\subsection{Relectura de metáforas y mitos: Un NUEVo metalenguaje}

Está claro que esta nueva visión fue respaldada por una relectura de las metáforas, de los mitos y de los términos empleados en la representación simbólica de la traducción. En concreto, se trataba de imágenes y palabras profundamente radicadas en la ideología sexista, cuyo objetivo era legitimar la subordinación del texto traducido al original como correlato de la relación asimétrica entre mujeres y hombres en la sociedad patriarcal. La violencia misógina de emblemas como la mujer esclava que hay que someter, las fases del proceso hermenéutico de Steiner o el mismo tropo de "les belles et infidèles" son el objeto de un estudio de Lori Chamberlain, que se propone indagar las vinculaciones del género a las metáforas traductivas con el objetivo de de-construirlas: "as feminist research from a variety of disciplines as shown, the opposition between productive and reproductive work organizes the way culture values work: this paradigm depicts originality or creativity in terms of paternity and authority, relegating the figure of female to a variety of secondary roles" (Chamberlain, 1988, p. 455).

Otro campo complementario desde el que cuestionar la concepción tradicional del fenómeno traductivo es el mito: en su Pandora's Tongues (2000), Karin Littau recupera la hipótesis de la caja de Pandora, descartada por Steiner y Derrida en favor de la torre de Babel, como explicación de la pluralidad lingüística. De hecho, mientras Babel alude a una unidad primigenia, a la caída de una cultura única e ideal, el caos de Pandora es capaz de acoger la multiplicidad femenina -en sentido irigarayano- y, por ende, de la traducción: "the many Pandora myths lend emphasis not to the impossibility of translation, but the impossibility of putting a stop of endless retranslation, in short, show us the serial nature of translation: there are always more translations, retranslations" (Littau, 2000, p. 32).

La redefinición simbólica del proceso traductivo se realiza también a través de un cambio en sus términos descriptivos. Para ello, las traductoras hacen eco al léxico empleado en el discurso androcéntrico, privilegiando los mecanismos de la parodia: por ejemplo, en su Re-belle et infidèle (1991), Susanne de Lotbinière-Harwood retoma el cliché de Gilles Ménage para rechazarlo con un juego de palabras y doblegarlo a su misma finalidad de resistencia al dominio patriarcal. Lo mismo hace Barbara Godard con sus "transformance" (Godard, 1989, p. 46) y "womanhandling" (Godard, 1989 , p. 50). El primero es un neologismo surgido de la fusión de "transformation" et "performance", para indicar una poética de trasformación crítica del texto, en contraste 
con la transparencia y la equivalencia de muchas teorías traductológicas masculinas. El segundo, en cambio, conmuta la agresividad del inglés "manhandling" (maltrato) por la "transgresión" de una feminización del texto que visibilice las intervenciones de las traductoras. En síntesis, la innovación de las feministas canadienses no se limitó al ámbito filosófico y epistemológico, sino que se repercutió también en el léxico y en los topoi de la teoría, hasta alcanzar una dimensión aún más tangible en la práctica.

\section{EL CAMBIO EN LA PRÁCTICA: ESTRATEGIAS APLICATIVAS}

La reflexión feminista sobre traducción se inscribe en la práctica a través de una serie de estrategias concretas, destinadas a evidenciar la identidad de las traductoras dentro de su trabajo. Como hemos bosquejado, su proyecto radica principalmente en la colaboración -platónica o real-con el/la autor@y, por lo tanto, el resultado coincide inevitablemente con la "polyphony of the translated text", como diría Godard (1989, p. 49). Pero, ¿cómo se intercalan las dos voces en el texto traducido, sin que la del autor@ prevalezca? Un primer recurso para reestablecer la autoría de la traductora consiste en la aposición de su firma: "the feminist translator immodestly flaunts her signature in italics, in footnotes- even in a preface" (Godard, 1989, p. 50). Lo que podría aparecer como un mero gesto simbólico, en realidad, contribuye a rediseñar el papel de la traductora como agente, plenamente dueña de su actividad. Esta estrategia encaja con la estética de la diferencia, con la voluntad de darle la palabra a lo femenino mediante el proceso productivo y re-creativo de la traducción. Otra posible modalidad consiste en lo que Von Flotow llama "supplementing" (1991, p. 74), una especie de integración, una sobretraducción ${ }^{11}$, que permitiría a la traductora subvertir la ideología patriarcal que rige el texto. De esta forma, lo implícito se manifiesta para ser corregido o, mejor dicho, de-construido en un nuevo texto, que impone la reflexión del público a partir de su sensación de desfamiliarización. Eso no quiere decir que la traductora pueda explicitar su lógica solo en los "huecos" del texto de partida: todo lo que no cabe en una simple modificación encuentra su explicitación en un espacio "metatextual", constituido por prólogos y notas al pie. Estas estrategias no solo permiten aclarar el porqué de las soluciones elegidas (compensando a veces la falta de comparación con el original que impide al público notar las intervenciones "políticas"), sino que construyen un perfil de la traductora, de sus ideas y de sus posturas. Los paratextos se convierten en el lugar de escucha de la subjetividad de la traductora, puesto que en ellos se nos cuentan los retos y las etapas de su recorrido, configurando así su presencia como la base de partida de todo el proceso. Por último, Von Flotow alude al "secuestro" o "hijacking" (1991, p. 78), que consiste en una apropiación del texto con el objetivo de reflejar la

11 Ya Benjamin utilizaba este término para describir el enriquecimiento del texto original a través de la traducción: "according to Benjamin the source text is supplemented by its translation, matured, developed, and given an afterlife" (Von Flotow, 1991, p. 75). 
intención política de la traductora, es decir su voluntad de visibilizar lo femenino. Dependiendo del contexto, la traductora puede llegar a introducir neologismos o a eliminar el neutro masculino, ya sea sustituyéndolo por el femenino genérico o recurriendo a perífrasis, barras, arrobas y guiones. A estas técnicas, podríamos sumar el "pacto especular" (Marie-France Dépêche, 2002, p. 19) que se da en los (raros) casos de intensa colaboración entre autor@y traductor@ hasta llegar a una verdadera coautoría. Cuando se logra aplicar esta estrategia, en realidad, se cumple del todo el afán igualitario de la traducción feminista: en la obra, aparecen juntos los nombres de 1@s dos autor@s, restableciendo incluso a nivel gráfico la paridad entre los dos trabajos, más allá de la tradicional noción de autoría. De acuerdo con la teoría deconstructivista, el texto se presenta finalmente como una multidimensionalidad impura, en que se entrecruzan muchos más escritos y cadenas de significación (intertextualidad).

Al final de este breve resumen de las principales estrategias desarrolladas por la escuela canadiense, cabe aclarar que:

el nivel de alteración o manipulación que implican las prácticas de traducción feminista no es necesariamente mayor del que implican otras prácticas no cuestionadas que se alzan en nombre de la objetividad, pero la diferencia básica entre unas y otras sí resulta capital. Mientras que las feministas son conscientes de las prácticas que implementan al tiempo que, de forma responsable, advierten y reconocen honestamente su adscripción ideológica y su postura subjetiva sobre una realidad que siempre es relativa, con la legitimidad que les otorga su compromiso de contribuir a la reforma lingüística para conducir al cambio social que supere la discriminación de género; las otras prácticas incuestionadas son invisibles, ocultan su intervención, camuflan su manipulación del texto dando a entender su "fidelidad" al original y, en definitiva, de forma deshonesta e inadvertida presentan su alteración como un hecho incontestable, objetivo y libre de ideología (Castro Vázquez, 2008, p. 298).

\section{RECEPCIÓN: LAS PIONERAS ESPAÑOLAS}

Con el objetivo de comprobar qué resonancia ha tenido la escuela canadiense en el contexto de difusión de este artículo, en este apartado vamos a trazar unos breves perfiles de algunas de las principales "pioneras" españolas. En este caso, se trata de las primeras traductoras y traductólogas que, acogiendo la propuesta de ultramar, empezaron a divulgar el nuevo pensamiento sobre traducción vinculado con el género. Cabe destacar que dicha teoría cruzó el charco con casi una década de retraso; por lo tanto, los primeros escritos acerca del tema en España remontan a finales de los años noventa. Una de las mediadoras más activas en este campo fue, sin duda, M. Carmen África Vidal Claramonte, quien, ya en 1995, empezó a plantear una visión deconstructivista y culturalista de la traducción, en línea con las premisas de las mismas traductoras canadienses. Sin embargo, hay que esperar 1998, para que, en su El 
futuro de la traducción: últimas teorías, nuevas aplicaciones, la autora presente los modelos teóricos y metodológicos de las feministas como los más cercanos a la perspectiva ética que propone para el futuro. Eso no quiere decir que acepte acríticamente las especulaciones de la escuela canadiense: en su De por qué no se puede traducir en femenino (1999), pone de relieve la contradicción de basarse explícitamente en una teoría como la posestructuralista, centrada en la crisis del sujeto, para luego reclamar una identidad fuerte y creadora del texto. Casi el mismo recorrido se encuentra en la obra de Pilar Godayol (2000): para posicionar lo femenino en traducción, ella también parte de un marco posestructuralista que aplica al desmonte y a la reconstrucción de los conceptos clave de las feministas canadienses. Su estudio llega incluso a ofrecer una muestra práctica de cómo subvertir el original a nivel ideológico, sin jamás evitar evidenciar las ambigüedades que eso conlleva, al ser la traducción un acto político. Además, Godayol incluye una pequeña historia internacional de algunas traductoras del pasado, contextualizando así la relatividad de las innovaciones teóricas sobre traducción y género. Su reflexión tiene un carácter tan abierto que podría ejemplificarse con la misma imagen de la frontera que ella recupera de las canadienses: se trata de un espacio de nadie, exento de juegos de poder y, por lo tanto, acogedor de la paradoja y de la multiplicidad de un verdadero diálogo. Nunca pone en entredicho la posibilidad de traducir también a los escritores: la teoría de Godayol, lejos de algunos excesos de las canadienses, apuesta por la reconciliación y abraza la incertidumbre, con una postura casi siempre intersticial y negociadora. Además, Godayol fue una de las primeras en centrarse en estudios de caso concretos (2006) y a abrirle paso a la investigación acerca de traducción y género en el contexto catalán (2011).

A parte de estas primeras especulaciones bastante aisladas, en general, los primeros pasos que se dieron en este campo en España apuntaban a contextualizar el fenómeno canadiense dentro de las demás teorías traductológicas, como en el caso de Hurtado Albir (2001) y Virgilio Moya (2004). Solo a finales de la primera década del siglo XXI, algunas de las traductoras que habían acogido la innovación de Canadá empezaron a cuestionar la importación de dichas teorías, analizando la forma con la que se habían implantado en el entorno académico ya existente, interactuando con otros avances y reflexiones del momento. Un primer núcleo de esta labor se encuentra en la tesis doctoral de Nuria Brufau Alvira (2009), dirigida por Vidal Claramonte, quien dedica un capítulo entero a la traducción feminista canadiense, formulando algunas dudas al respecto y, sobre todo, abarcando el tema de su transposición y recepción en España. Según ella,

en España, el gran reconocimiento del interés de la traducción en las cuestiones de género, en un intento por distanciarse de la mera crítica y comentario del caso canadiense, llegó en 2002, con la celebración en Valencia del "Primer Seminario Internacional sobre Género y Lenguaje" cuyo tema era "El género de la traducción- 
La traduccióń del género", y que fue auspiciado por Josë Santaemilia, quien también se encargó de editar las actas Género, lenguaje y traducción, publicadas en 2003 (Brufau Alvira, 2009, p. 411).

Desde entonces, el estado de la cuestión ha evolucionado considerablemente: ya al cabo de pocos años, Brufau Alvira actualizó su investigación con un artículo (2011) dedicado a extrapolar las tendencias contemporáneas de las últimas contribuciones publicadas en España. El estudio, esta vez, se enmarcaba en una recopilación de trabajos -muy variados desde el punto de vista geográfico- cuyo objetivo era demonstrar el crecimiento del interés académico acerca del tema (Santaemilia \& Von Flotow, 2011). A la luz de los resultados obtenidos por la estudiosa, el pronóstico para el futuro es el de una cada vez más intensa cooperación entre el feminismo y la traducción para la mejora de la igualdad.

\section{ALGUNAS CONCLUSIONES}

Resumiendo, el alcance de las teorías feministas sobre traducción no se puede medir sin tener en cuenta su doble logro: no se trata solo de revindicar una nueva concepción de la traducción y de 1@s traductor@s que sea más inclusiva, sino de fomentar un cambio también en la práctica, abriéndole paso a una nueva forma de manipular el texto de carácter ideológico. Gracias a las canadienses, las traductoras se han vuelto figuras empeñadas en lo político: su trabajo puede influir en la recepción de una obra extranjera o dar a conocer textos novedosos de mujeres que habían sido descuidadas o "corregidas" en su enfrentamiento hacia el patriarcado. Además, la capacidad de la traducción de traspasar fronteras permitió a la escuela canadiense expandirse más allá de sus límites geográficos: hemos visto que a partir de los años noventa, algunas teóricas españolas, impulsadas por las feministas de ultramar, ya habían empezado a plantearse nuevos modelos de traducción y, sobre todo, a cuestionar y readaptar el pensamiento de sus compañeras. La capacidad innovadora de estas mujeres transciende su campo de investigación para llegar a una propuesta de empoderamiento más amplia: si miramos a la traducción como a una herramienta de construcción de identidades, está claro que declarar una postura explícita en un texto equivale a una forma de posicionamiento subjetivo en la sociedad. En otras palabras, se supone que un cambio en las relaciones de poder a nivel lingüístico y textual favorezca una evolución de la mentalidad colectiva. Mientras la incorporación del feminismo a la traducción está tomando pie paulatinamente, gracias a los programas universitarios de formación y a la divulgación cultural, el legado de las canadienses ya ha desembocado en una red sororal de estudiosas y traductoras determinadas a volver a apropiarse de su trabajo. 


\section{REFERENCIAS BIBLIOGRÁFICAS}

Agorni, Mirella (2014). Translating Italy for the Eighteenth Century: British Women, Translation and Travel Writing (1739-1797). Londres- Nueva York: Routledge.

Brufau Alvira, Nuria (2009). Traducción y género: propuestas para nuevas éticas de la traducción en la era del feminismo transnacional [tesis doctoral, Universidad de Salamanca]. https://gredos.usal.es/bitstream/handle/10366/76219/DTI_ BrufauAlviraN_TraduccionyGenero.pdf?sequence=1\&isAllowed=y [consultado el $12 / 11 / 2020]$.

Brufau Alvira, Nuria (2011). Traducción y género: el estado de la cuestión en España. MonTI. Monografías de Traducción e Interpretación, 3, 181-207, https://rua.ua.es/ dspace/bitstream/10045/21612/1/MonTI_3_08.pdf [consultado el 03/11/2020].

Castro Vázquez, Olga (2008). Género y traducción: elementos discursivos para una reescritura feminista. Lectora, 14, 285-301, https://www.researchgate.net/ publication/46733857_Genero_y_traduccion_elementos_discursivos_para_una_ reescritura_feminista [consultado el 26/10/2020].

Chamberlain, Lori (1988). Gender and the Metaphorics of Translation. Signs 13(3), 454472, https://www.jstor.org/stable/3174168?seq=1 [consultado el 12/10/2020].

Chamberlain, Lori (1998). Gender Metaphorics in Translation. En Mona Baker (Ed.), Routledge Encyclopedia of Translation Studies (pp. 93-96). Londres-Nueva York: Routledge.

Delisle, Jean (2002). Portraits des traductrices. Ottawa: Presse de l'Université d'Ottawa.

Dépêche, Marie-France (2002). As traduções subversivas feministas ontem e hoje. Labrys, Estudos Feministas, 1-2, 1-26.

Derrida, Jacques (1986). La loi du genre. En Jacques Derrida, Parages (pp. 251-287). París: Galilée.

Even-Zohar, Itamar (1979). Polysystem theory. Poetics Today, 1-2, 287-310.

Godard, Barbara (1985). The translator as she: The relationship between writer and translator. En A. Dybikowski, V. Freeman, D. Marlatt, B. Pulling, y B. Warland (Eds.), In the feminine: Women and words/Les femmes et les mots (pp. 193-198). Edmonton, AB: Longspoon.

Godard, Barbara (1989). Theorizing Feminist Discourse/ Translation. Tessera, 6, 42-53. DOI: https://doi.org/10.25071/1923-9408.23583, https://tessera.journals.yorku.ca/ index.php/tessera/article/view/23583/21792 [consultado el 16/11/2020].

Godayol i Nogué, María Pilar (2000). Espais de Frontera: Genere i Traducció. Barcelona: Eumo, 
Godayol i Nogué, María Pilar (2006). Helena Valentí, fúria i traducció. Quaderns. Revista de traducció, 13, 87-93, https://www.raco.cat/index.php/QuadernsTraduccio/ article/view/51664 [consultado el 18/11/2020].

Godayol i Nogué, María Pilar (2008). Derrida y la teoría de la traducción en femenino. DeSignis, 12, 67-74.

Godayol i Nogué, María Pilar (2011). Gènere i traducció en català. Bases arqueològiques per a un estat de la qüestió. MonTI, Monografías de Traducción e Interpretación, 3, 5373, https://rua.ua.es/dspace/bitstream/10045/21604/1/MonTI_3_03.pdf [consultado el 17/11/2020].

Guerra-Cunningham, Lucía (1995). La mujer fragmentada: historias de un signo. Santiago de Chile: Editorial Cuarto Propio.

Guerrero Alcalde, Jennifer (2017). El papel de la mujer en la traducción española contemporánea (1850-1950) [tesis doctoral, Universitat Autònoma de Barcelona] https://ddd.uab.cat/pub/tfg/2017/tfg_67168/TFG_2016-17_FTI_GuerreroAlcalde. pdf [consultado el 10/11/2020].

Hurtado Albir, Amparo. (2001). Traducción y traductología. Introducción a la traductología. Madrid: Cátedra,

Lefevere, André (1992). Translation, Rewriting and the Manipulation of Literary Fame. Londres-Nueva York: Routledge.

Lerner, Gerda (1990). La creación del patriarcado. Barcelona: Editorial Crítica.

Littau, Karin (2000). Pandora's tongues, TTR, 13(1), 21-35. DOI: 10.7202/037391ar

Lotbinière-Harwood, Susanne (1991). Re-belle et infidèle : la traduction comme pratique de réécriture au féminin/ The Body Bilingual: Translation as a Rewriting in the Feminine. Montréal: Women's Press/Éditions du remue-ménage.

Mezei, Kathy (2013). Il dialogo nella traduzione letteraria contemporanea. En Debora Saidero (Ed.), La traduzione femminista in Canada (pp. 17-35). Udine: Editrice Universitaria Udinese.

Mills Sara \& Mullany Louise (2011). Language, Gender and Feminism. Londres-Nueva York: Routledge.

Moya, Virgilio (2004). La selva de la traducción. Teorías traductológicas contemporáneas. Madrid: Cátedra.

Pieretti, Marie-Pascale (2002). Women Writers and Translation in Eighteenth-Century. The French Review, 75(3): 474-488. DOI: 10.2307/3132846, https://www.jstor.org/ stable/3132846?seq=1 [consultado el 14/11/2020].

Reiss, Katharina \& Vermeer Hans Josef (1984). Grundlegung einer allgemeinen Translationstheorie. Tübingen: Niemeyer. 
Romero López, Dolores (2015). Mujeres traductoras en la Edad de Plata (1868-1939): identidad moderna y affidamento. Hermēneus. Revista de Traducción e Interpretación, 17, 179-207 https://www.researchgate.net/publication/299454290_Mujeres_ traductoras_en_la_Edad_de_Plata_1868-1936_Identidad_moderna_y_affidamento [consultado el 16/11/2020].

Romero López, Dolores (Ed.). (2016). Retratos de traductoras en la Edad de Plata. Madrid: Escolar y Mayo.

Santaemilia, José y Von Flotow, Luise (Eds.) (2011). Woman And Translation: Geographies, Voices And Identities. MonTI, 3 https://rua.ua.es/dspace/ bitstream/10045/27170/1/MonTI_3.pdf [consultado el 19/11/2020].

Simon, Sherry (1996). Gender in Translation. Cultural Identity and the Politics of Transmission. Londres-Nueva York: Routledge.

Snell-Hornby, Mary (1990). Linguistic transcoding or cultural transfer: a critique of translation theory in Germany. En Susan Bassnett y André Lefevere (Eds.), Translation, History and Culture (pp. 79-86). Londres-Nueva York: Routledge.

Toury, Gideon (1995). Descriptive Translation Studies - and Beyond. Amsterdam / Philadelphia: John Benjamins Publishing Company.

Venuti, Lawrence (1995). The Translator's Invisibility. A History of Translation. LondresNueva York: Routledge.

Vidal Claramonte, María del Carmen África (1995). Traducción, manipulación, desconstrucción. Salamanca: Ediciones Colegio de España (Colección Biblioteca Filológica de Salamanca).

Vidal Claramonte, María del Carmen África (1998). El futuro de la traducción: últimas teorías, nuevas aplicaciones. Valencia: Alfons el Magnànim.

Vidal Claramonte, María del Carmen África (1999). De por qué no se puede traducir en femenino. En Miguel Ángel Vega Cernuda y Rafael Martín-Gaitero (Eds.), Lengua y cultura. Estudios en torno a la traducción. Actas del VII Encuentro complutense en torno a la traducción (II) (pp. 229-232). Madrid: Editorial complutense https:// cvc.cervantes.es/lengua/iulmyt/pdf/lengua_cultura/25_vidal.pdf [consultado el 13/11/2020].

Von Flotow, Luise (1991). Feminist Translation: Contexts, Practices and Theories, TTR, 42, 69-84. DOI: 10.7202/037094ar, https://www.researchgate.net/ publication/238728257_Feminist_Translation_Contexts_Practices_and_Theories [consultado el 06/11/2020].

Von Flotow, Luise (1997). Translation and Gender: Translating in the "Era of Feminism". Manchester: St. Jerome. 
Valentina Tomassini 